\title{
Le management des connaissances : des concepts aux expériences, des expériences à la méthode
}

Jean-Yves Prax

\section{(2) OpenEdition}

\section{Journals}

Édition électronique

URL : http://journals.openedition.org/communicationorganisation/2024

DOI : 10.4000/communicationorganisation.2024

ISSN : $1775-3546$

\section{Éditeur}

Presses universitaires de Bordeaux

Édition imprimée

Date de publication : 1 mai 1998

ISSN : 1168-5549

\section{Référence électronique}

Jean-Yves Prax, "Le management des connaissances : des concepts aux expériences, des

expériences à la méthode », Communication et organisation [En ligne], 13 | 1998, mis en ligne le 26 mars 2012, consulté le 21 décembre 2020. URL : http://journals.openedition.org/

communicationorganisation/2024 ; DOI : https://doi.org/10.4000/communicationorganisation.2024

Ce document a été généré automatiquement le 21 décembre 2020.

(C) Presses universitaires de Bordeaux 


\section{Le management des connaissances : des concepts aux expériences, des expériences à la méthode}

Jean-Yves Prax

1 Une nouvelle forme d'organisation émerge, fondée sur la connaissance et l'intelligence collectives et non plus seulement sur les moyens et les structures. Elle correspond pour l'entreprise contemporaine à une nécessité d'adaptation ultra-réactive, permanente et répartie sur l'ensemble des acteurs, face à un environnement complexe, incertain et hautement concurrentiel.

2 Sur ce terrain fertile fleurissent :

3 - les technologies : groupware, GED et workflow, édition électronique Internet/Intranet et CD-ROM, agents, modélisation des experts...

4 - les concepts: la capitalisation, le management et la gestion stratégique des connaissances, l'intelligence collective, l'entreprise apprenante, le knowledge management...

5 - les dispositifs : knowledge ressource center, best practices, réseaux de compétence, management par projet, par processus, veille technologique, etc..

6 En face de ce foisonnement sans limite, le constat sur l'utilisation effective des bases de connaissance dans l'entreprise est plutôt mitigé : résistances culturelles (ou politiques) au changement? Difficultés liées à l'immaturité des outils?

7 Inadaptation des règles économiques à la production immatérielle? Difficultés méthodologiques ou conceptuelles?

\section{Première partie : la connaissance organisationnelle}

Depuis toujours, les organisations ont cherché à bâtir une interprétation « objective et formelle » de leur environnement avec l'espoir d'en réduire la complexité et l'incertitude. 


\section{Les systèmes de représentation} réalité, un objet universellement partagé et la recherche d'une vérité unique. Dans cette logique, le but du scientifique était de découvrir « le plan de câblage de l'univers », puis de le partager sous forme d'universaux en tout genre: axiomes, théorèmes, règles et méthodes, croyances. dire réductibles par l'analyse à une somme d'éléments premiers, dont le sens est connu par tous. présentation individuelle de l'observateur, par sa construction mentale, variable en fonction de sa culture, de ses intentions ou de son projet, et de son contexte d'observation. 
relations, analogies, correspondances, et tout un appareillage de concepts élaborés par le sujet lui-même.

Figure 1 : composants du système de représentation (d'après $D$. Genelot ${ }^{1}$ )

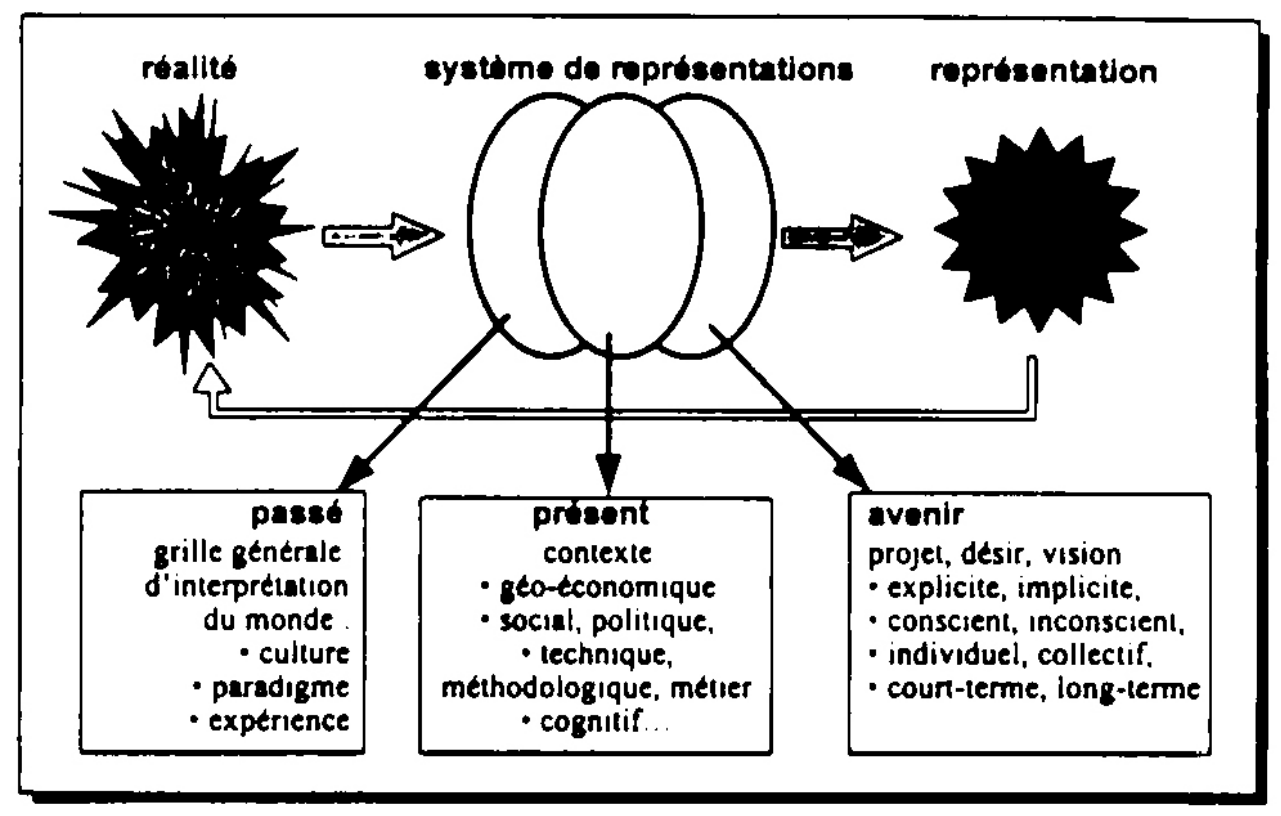

Une fois admis que la subjectivité gouverne l'ensemble des représentations individuelles d'un système, on conçoit que le processus de construction collective d'une représentation passe nécessairement par une étape de mise en commun des perceptions, de confrontation, de négociation et de délibération de ces différentes subjectivités.

Cela concerne en premier lieu le monde de l'entreprise, et à tout son pilotage stratégique, qui prend sa source dans la vision intersubjective des différents acteurs. réalité système de représentations représentation

31 Cette étape est nécessaire à la construction d'un sens commun, au passage d'une somme de connaissances individuelles à une connaissance collective. Cette étape est aussi celle par laquelle la connaissance explicite collective génère du tacite pour chaque individu.

\section{Connaissance tacite et connaissance explicite}

Dans ses travaux, Nonaka ${ }^{2}$ distingue quatre états de connaissance: une connaissance tacite individuelle, explicite individuelle, explicite collective et tacite collective. Il montre que le développement des savoirs dans l'organisation implique la transmission entre les individus et il décrit la dynamique de transformation entre ces différents états :

33 - du tacite vers le tacite : socialisation

34 - du tacite vers l'explicite : formalisation

35 - de l'explicite vers le tacite : intériorisation

36 - de l'explicite vers l'explicite : combinaison 


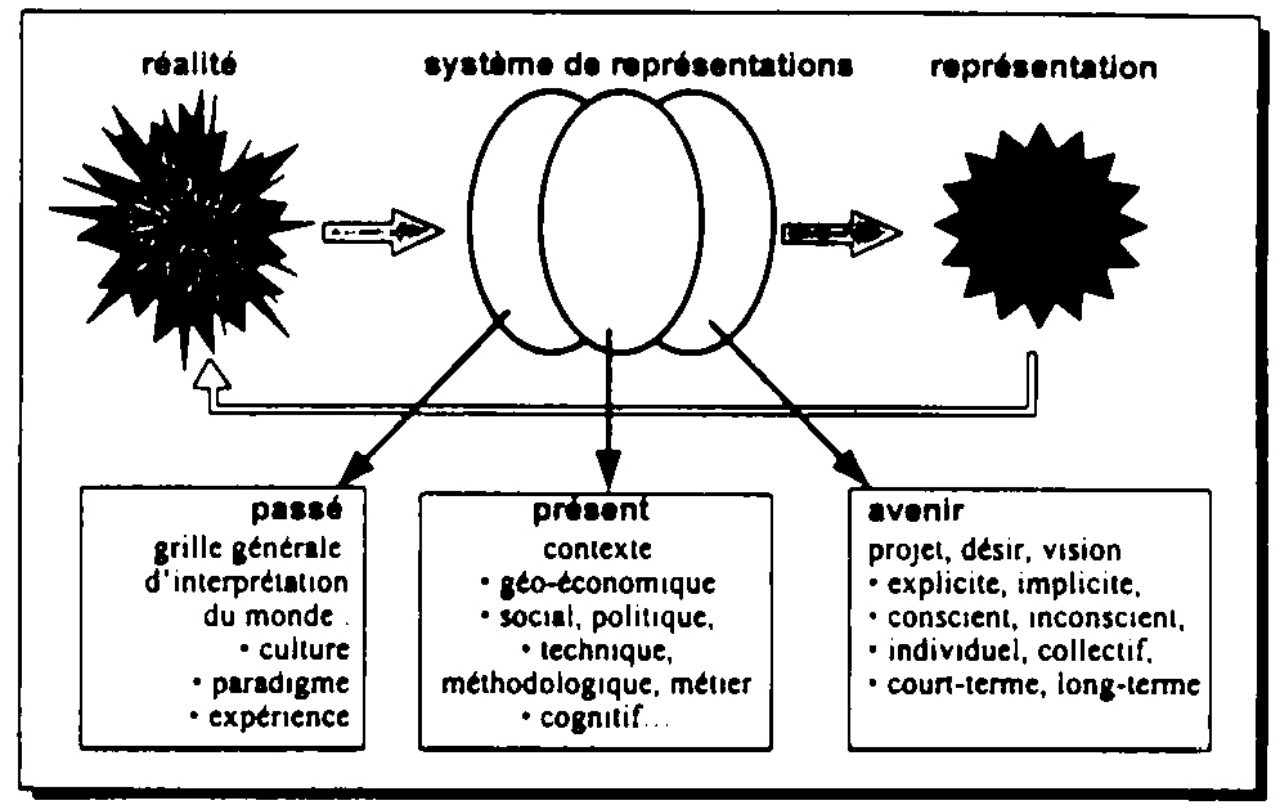

\section{connaissances explicites qui sont combinées, rapprochées pour produire, par induction,} déduction, hybridation, des connaissances nouvelles.

\section{Le langage et l'action}

41 Si nous voulons adopter une approche collective de la connaissance, nous sommes amenés à nous situer dans un espace de médiation, qui s'appuie sur le langage, dans le domaine des savoirs explicites, et sur l'action dans le domaine des savoir-faire.

Mais le langage n'est pas un vecteur de communication parfait. Contrairement à ce qu'affirment les théories shannoniennes de la communication, il ne suffit pas d'un input, d'un output et d'un tuyau pour qu'une connaissance passe d'un individu à un autre.

D'abord le langage est multi-plan : trop d'abstraction et de globalisation dans le langage stérilise le contenu, trop de particularisme et de spécificité entraîne du bruit et nuit à la compréhension; si les acteurs veulent communiquer efficacement, ils doivent se situer sur le même plan.

Ensuite, avec la sémantique, le langage porte en lui-même toute la subjectivité des acteurs. En effet, si le mot, comme symbole collectif, appartient à la communauté 
linguistique et sémantique, le sens qu'il recouvre est purement individuel car il est intimement lié à l'expérience et à l'environnement cognitif dans lequel se place l'individu. Dans une conversation, deux interlocuteurs peuvent arriver à partager des mêmes points de vue s'ils établissent un processus de coopération: écoute active, participation, questionnement, adaptation sémantique, feed-back, reformulation.

Cette composante sémantique est bien entendu capitale pour la mise au point des outils de capitalisation de la connaissance car elle sous-tend la question de la pertinence des descripteurs, des mots-clés, et du thésaurus et de l'articulation entre les dispositifs " semi-conversationnels », comme le groupware, et les dispositifs « référentiels », comme la Gestion Electronique Documentaire.

C'est le projet d'action qui constitue le moteur de l'apprentissage organisationnel ; c'est le projet qui focalise l'attention sélective (la veille) du sujet; c'est à travers l'action que les différents acteurs vont fabriquer un sens partagé aux différentes données dont ils disposent, se créer une sémantique commune, parfois sub-langagière. L'action favorise la création et l'ancrage de connaissances « collectivisées » et principalement tacites.

\section{L'iceberg relationnel}

47 À ce stade, il est nécessaire d'évoquer le statut de la "confiance " dans l'univers relationnel des organisations et des acteurs.

Le tacite est source et reconnaissance de la confiance entre les acteurs. En passant du tacite au formel, on "libère l'attribution de sens de ses ambiguïtés »", et, ce faisant, soit on évite ou on brise la relation de confiance entre les individus (c'est le cas du contrat juridique), soit au contraire, on force le renouvellement de la confiance sur un niveau supérieur à celui des instances formalisées (c'est le cas de la charte, ou du credo).

Figure 3 : l'iceberg relationnel

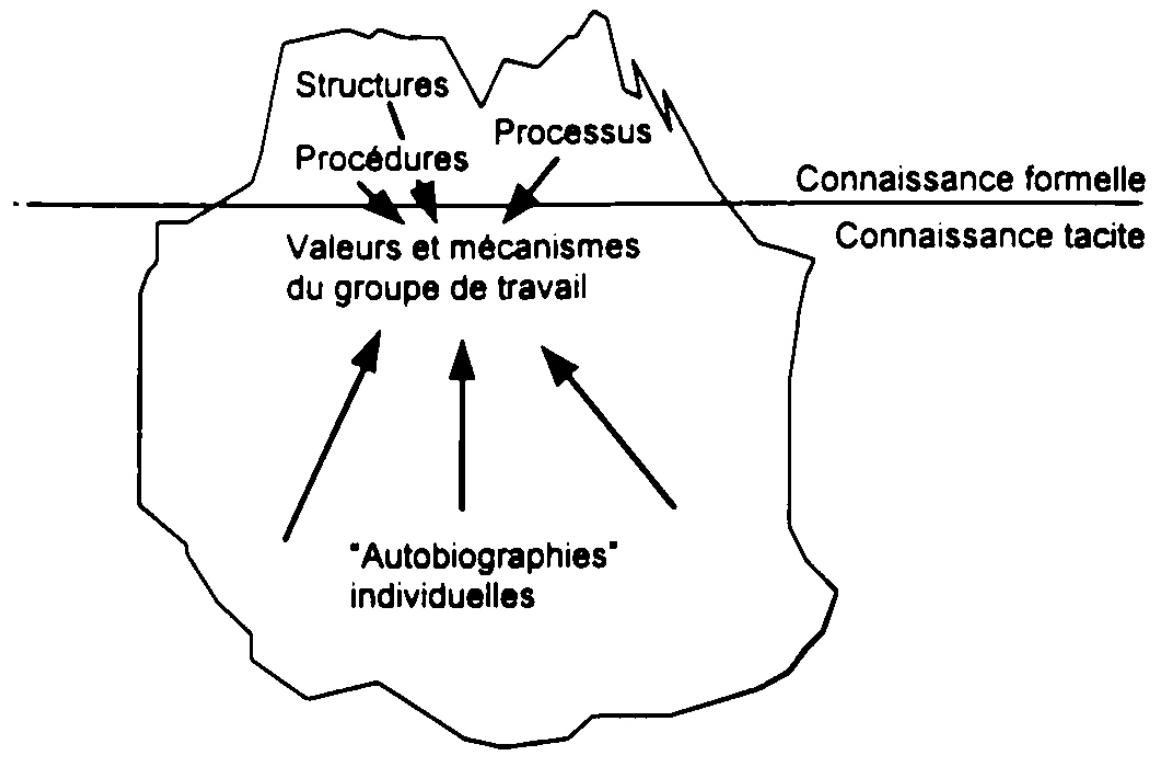

Partant de ces hypothèses, la politique relationnelle d'une entreprise, ou d'une communauté d'acteurs au sein d'une entreprise, n'obéit à aucune règle ou recette globale. 

fait le groupe, et ce style va totalement conditionner la forme de l'apprentissage collectif. Or ce style hérite de valeurs tacites, de variables cachées, d'habitudes, de comportements et d'interactions entre les personnalités, très difficiles à analyser et à changer.

\section{La capitalisation} connaissance collective ; seulement ce capital est rarement disponible instantanément et dans un contexte pertinent. De surcroît, l'appropriation de cette connaissance peut rarement se faire autrement que par questionnement direct des acteurs, «ceux qui savent ».

52 La capitalisation désigne le passage d'une mémoire de travail à une mémoire organisationnelle. La mémoire organisationnelle désigne le capital de connaissance accessible indépendamment des acteurs qui l'ont créée. C'est un patrimoine pour l'entreprise qui doit perdurer aux acteurs.

53 Sans cet effort de capitalisation, la connaissance collective n'existe pas: elle reste virtuelle et se réduit à une somme d'expériences isolées.

La connaissance étant fortement " subjective », on devine que la difficulté principale de la capitalisation de connaissance collective réside dans le choix des critères descripteurs qui devront être pertinents pour tout le monde, en tous lieux et en tout temps, sans pour autant trop appauvrir ou généraliser le contenu.

La production de sens collectif s'établit dans un contexte aux contours mouvants, avec une tendance spontanée à la personnification, à la contextualisation, à la temporalisation. On raccroche le sens à l'acteur, à la situation ou à l'événement qui la généré.

Toute démarche contraire (décontexualisation, objectivation...) va fabriquer de la connaissance organisationnelle abstraite, généraliste, procédurale, fossilisée. La difficulté réside dans l'articulation de ces deux tendances antagonistes.

\section{L'individu, le groupe, l'entreprise}

57 En résumé, il apparaît clairement à travers ces développements qu'une distinction majeure doit être faite entre le niveau du groupe (team level) et le niveau de la firme (corporate level).

La constitution d'une connaissance collective au niveau du groupe va se faire par une série d'interactions entre le tacite et l'explicite et entre l'explicite et le tacite; ces interactions vont pouvoir s'appuyer sur les contacts physiques entre les individus: identification mutuelle, rôles, postures, processus de confiance, délibération orale, action et projet commun, langage partagé, responsabilité partagée, etc.. Dans ce domaine, les dispositifs de socialisation: cafétéria, open-space, tutorat, réunion d'équipe... seront déterminants; ils pourront être efficacement relayés ou appuyés par des outils de type groupware, visioconférence, Intranet.

59 Mais autre chose est la connaissance organisationnelle, au niveau de la firme, c'est-à-dire ce patrimoine commun et partagé par des acteurs géographiquement dispersés et qui ne se connaissent pas. Si la délibération à deux ou à dix personnes est permise, à quarante elle devient difficile, à quatre mille elle est utopique !

Communication et organisation, 13 | 1998 
60 Le niveau de la firme impose un système d'échange qui va donc forcément au delà du périmètre où les acteurs se connaissent et se rencontrent. Il ne s'agit pas pour autant de retourner dans un système pyramidal où les relais informationnels top-down sont effectués par le middle-management, sans rétroaction; ni dans un système cloisonné fait d'une juxtaposition de cellules hyperspécialisées.

61 Nous devons inventer de nouveaux dispositifs qui abandonnent les anciens formalismes pour s'appuyer sur deux facteurs structurants; le projet, qui est le moteur de l'action et de la convergence entre les acteurs, et les méthodologies qui assurent la cohérence dans l'action. La première conséquence de ce mode de pilotage est que le savoir et l'intelligence ne sont plus centralisés mais répartis sur l'ensemble de l'organisation; la deuxième est que la fluidité des échanges d'informations et des connaissances s'en trouve accrue, un langage commun et une culture commune sont créés.

Au principe moteur basé sur l'articulation des moyens se substitue une articulation des compétences et des savoir-faire. La fonction d'animation est déterminante dans la stabilisation de cette production d'actifs immatériels.

\section{Deuxième partie : les dispositifs de l'ingénierie de la connaissance}

63 Nos expériences en entreprise ont montré que la conduite du changement supposait une action conjointe à trois niveaux :

64 1. celui de l'outil (boucle technique), apte à apporter de nouveaux modes de travail,

65 2. celui du management (boucle organisationnelle), apte à piloter les usages et des processus d'action,

66 3. celui de la stratégie (boucle stratégique), apte à changer les représentations, la culture, et les valeurs directrices sous-jacentes aux modes de travail.

Dans la pratique, ces trois boucles ne sont pas séparées, elles s'imbriquent et se nourrissent mutuellement; il est primordial de traiter ces trois boucles dans leurs interactions et pas seulement de façon séquentielle.

L'entrée des acteurs dans le projet se fait par la boucle 2, celle du contenu, des chantiers et leur animation, des modes d'action, des usages et de l'organisation des acteurs... C'est le pilotage organisationnel du projet, c'est le moteur de la motivation du groupe et de l'identification mutuelle des compétences.

69 Pour mener à bien ce projet, compte tenu de la nouvelle donne : dispersion des acteurs, délais incompatibles avec les emplois du temps, nécessité de capitaliser les retours d'expérience, le projet d'action (boucle 2) décide de s'appuyer sur un outil nouveau: les NTIC.

70 Le pilotage technique vise à une appropriation qui fait oublier l'outil au profit du projet d'action défini dans la boucle 2. Il change les habitudes de travail.

71 Grâce à la focalisation des acteurs sur les boucles 1 et 2, et grâce à l'introduction de ces nouveaux modes de travail, on vise ainsi les finalités stratégiques, qui sont parfois des enjeux cachés ou des valeurs sous-jacentes : par exemple apprendre aux gens à travailler ensemble... 


\section{Le niveau outil : les NTIC au service de l'ingénierie de la connaissance}

72 En dépit de ses promesses et de ses efforts, en particulier dans le domaine de l'intelligence artificielle, l'informatique classique (que l'on peut qualifier d'analytique) n'a pas su jouer le rôle attendu dans l'ingénierie de la connaissance, car elle procède d'une rationalité qui est profondément incompatible avec l'inépuisable complexité du concept de connaissance :

73 - l'informatique ne gère que des données structurées selon des modèles préalables de représentations, alors que la connaissance collective se construit dans d'action et avec un langage sémantisé, c'est-à-dire portant en lui toute la charge subjective, affective et culturelle des individus,

74 - l'informatique traite les données selon des algorithmes prédéfinis par l'analyse, par rapport à une certaine idée du mai, alors que l'organisation, face à un environnement complexe et imprévisible, construit en permanence une représentation collective de la situation de façon à optimiser ses résultats : elle cherche la meilleure solution au lieu de chercher la vraie solution.

75 - l'informatique transporte les données sur un mode de communication purement physique, sur un modèle input-output, alors que la communication entre les hommes suppose des processus psychologiques de "couplage participatif» beaucoup plus complexes, dans lesquels l'autobiographie de l'émetteur et du récepteur, leur projet, leur modèle respectif de représentation jouent un rôle aussi important que les tuyaux et les bits.

\section{Les facteurs de complexité de l'informatique}

Porté par une très forte croissance, à la fois en valeur absolue et en valeur relative, le marché des constructeurs de puces, d'ordinateurs, de mémoires, de périphériques, de réseaux, a consenti, pendant plus de dix ans, des efforts de recherche et développement hors normes, dépassant parfois $20 \% \mathrm{du}$ C.A. Ces investissements se sont traduits par une évolution considérable des performances et des prix, et donc du parc installé, qui a surpris et continue encore à surprendre toutes les prévisions.

77 Cette explosion électronique a eu comme premier effet secondaire une croissance exponentielle, non contrôlée, du volume des informations publiées. Entre 1970 et 1985, les rapports scientifiques accessibles sur bases de données sont passés de cinquante-deux millions à deux milliards et croissent de cinq mille par jour. Cette augmentation de productivité éditoriale n'a pas que du bon, et l'expression « taylorisme des cols blancs » a été prononcée; par ailleurs, il fut rapidement hors de portée de la majorité des organisations de contrôler leurs propres flux informationnels, d'enrayer l'hémorragie éditoriale, avant même d'oser parler de capitalisation ou d'apprentissage organisationnel...

Le coût de gestion (production, recherche, classement, tri...) de ces montages d'information est estimé entre 3 et $8 \%$ du chiffre d'affaires et la tendance est à la hausse.

79 Les tentatives d'archivage documentaire des années 80 ont été décevantes, et le mythe du « zéro-papier » s'est vite révélé une utopie à une époque où « les ordinateurs personnels, armés de leurs imprimantes laser, ne faisaient que le bonheur des marchands de pâte à papier » 


\section{Témoignage des utilisateurs : «Trop d'info tue l'info »}

80 Enoncé comme cela, le constat est (malheureusement déjà) une banalité: l'homme contemporain subit passivement le raz-de-marée informationnel ; l'information qui était censée l'aider dans le processus de décision, d'action, devient un facteur de complexité supplémentaire.

81 Nos récentes missions dans de grandes entreprises nous ont permis d'analyser plus finement cette problématique et d'en tirer les premiers enseignements, que l'on peut résumer par le tableau suivant :

Tableau 1 : Analyse des principaux facteurs de complexité liés au traitement de l'information

\begin{tabular}{|c|c|c|}
\hline $\begin{array}{c}\text { Facteurs } \\
\text { de complexité }\end{array}$ & $\begin{array}{l}\text { "la voix" } \\
\text { de l'utilisateur }\end{array}$ & Levier d'action \\
\hline \multirow{3}{*}{$\begin{array}{l}\text { Le raz-de-marée } \\
\text { informationnel }\end{array}$} & $\begin{array}{l}\text { le volume de } \\
\text { linformation publiée }\end{array}$ & $\begin{array}{l}\text { - séparer le contenu du } \\
\text { repérage } \\
\text { - améliorer la qualité } \\
\text { rédactionnelle } \\
\text { - mettre en regard l'offre et la } \\
\text { demande }\end{array}$ \\
\hline & $\begin{array}{l}\text { le statut du document } \\
\text { électronique }\end{array}$ & $\begin{array}{l}\text { - e-mail : média oral ou écrit? } \\
\text { - signature électronique }\end{array}$ \\
\hline & $\begin{array}{l}\text { des règles de fonctionnement } \\
\text { des communautés virtuelles }\end{array}$ & $\begin{array}{l}\text { - certifier les sites et forums } \\
\text { - clóture des forums } \\
\text { - séparer lhomme de la } \\
\text { fonction }\end{array}$ \\
\hline \multirow{4}{*}{$\begin{array}{c}\text { L'interface } \\
\text { homme-machine }\end{array}$} & $\begin{array}{l}\text { Difficulté liée à } \\
\text { l'apprentissage } \\
\text { d'applications multiples }\end{array}$ & $\begin{array}{l}\text { - un outil fédérateur } \\
\text { d'interface }\end{array}$ \\
\hline & $\begin{array}{l}\text { Fossé entre ceux qui } \\
\text { maitrisent et ceux qui ne } \\
\text { maitrisent pas }\end{array}$ & $\begin{array}{l}\text { - démystifier l'outil, favoriser } \\
\text { l'appropriation }\end{array}$ \\
\hline & $\begin{array}{l}\text { syndrome de } \\
\text { "l'employement-killer" }\end{array}$ & - conception participative \\
\hline & $\begin{array}{l}\text { effroi du vocabulaire branché } \\
\text { des cybemautes }\end{array}$ & - glossaire en ligne \\
\hline $\begin{array}{l}\text { L'évolution } \\
\text { des compétences }\end{array}$ & $\begin{array}{l}\text { Mobiliser les connaissances et } \\
\text { l'intelligence pour formuler } \\
\text { des réponses inédites a des } \\
\text { problemes nouveaux et } \\
\text { imprévisibles }\end{array}$ & $\begin{array}{l}\text { - construire des } \\
\text { représentations finalisantes } \\
\text { des situations coomplexes }\end{array}$ \\
\hline \multirow[t]{2}{*}{$\begin{array}{l}\text { L'économie } \\
\text { de la connaissance }\end{array}$} & $\begin{array}{l}\text { Un cadre passe plus de } 60 \% \\
\text { de son temps sur le S.I. qui } \\
\text { r'est pas son caeur de métier }\end{array}$ & $\begin{array}{l}\text { - La capitalisation de } \\
\text { l'information fait partie des } \\
\text { missions et des objectifs } \\
\text { individuels }\end{array}$ \\
\hline & $\begin{array}{l}\text { Un cadre ne mesure pas le } \\
\text { retour sur investissement }\end{array}$ & $\begin{array}{l}\text { - choix de l'échelle de temps } \\
\text { du Retour sur } \\
\text { Investissement }\end{array}$ \\
\hline
\end{tabular}

\section{Les Nouvelles Technologies de l'Information et de la Communication}

Les NTIC dont il est question ici apportent une réponse nouvelle à notre problématique : au lieu de chercher à gérer, traiter et diffuser des données, elles se placent comme facilitateur de la communication, de la coordination et de la coopération entres les acteurs, et par conséquent du processus de création de sens ; au lieu de chercher à stocker l'information, elles mettent en place des dispositifs d'incitation à la création de connaissance et d'enrichissement des savoirs ; au lieu de chercher à diffuser, elles tissent un réseau non linéaire et sans cesse renouvelé de liens informationnels co-construits par les auteurs-lecteurs et les lecteurs-auteurs.

À partir de ses travaux et de ses expériences, l'auteur propose un modèle de NTIC pour l'ingénierie de la connaissance collective ${ }^{5}$ en trois niveaux : 


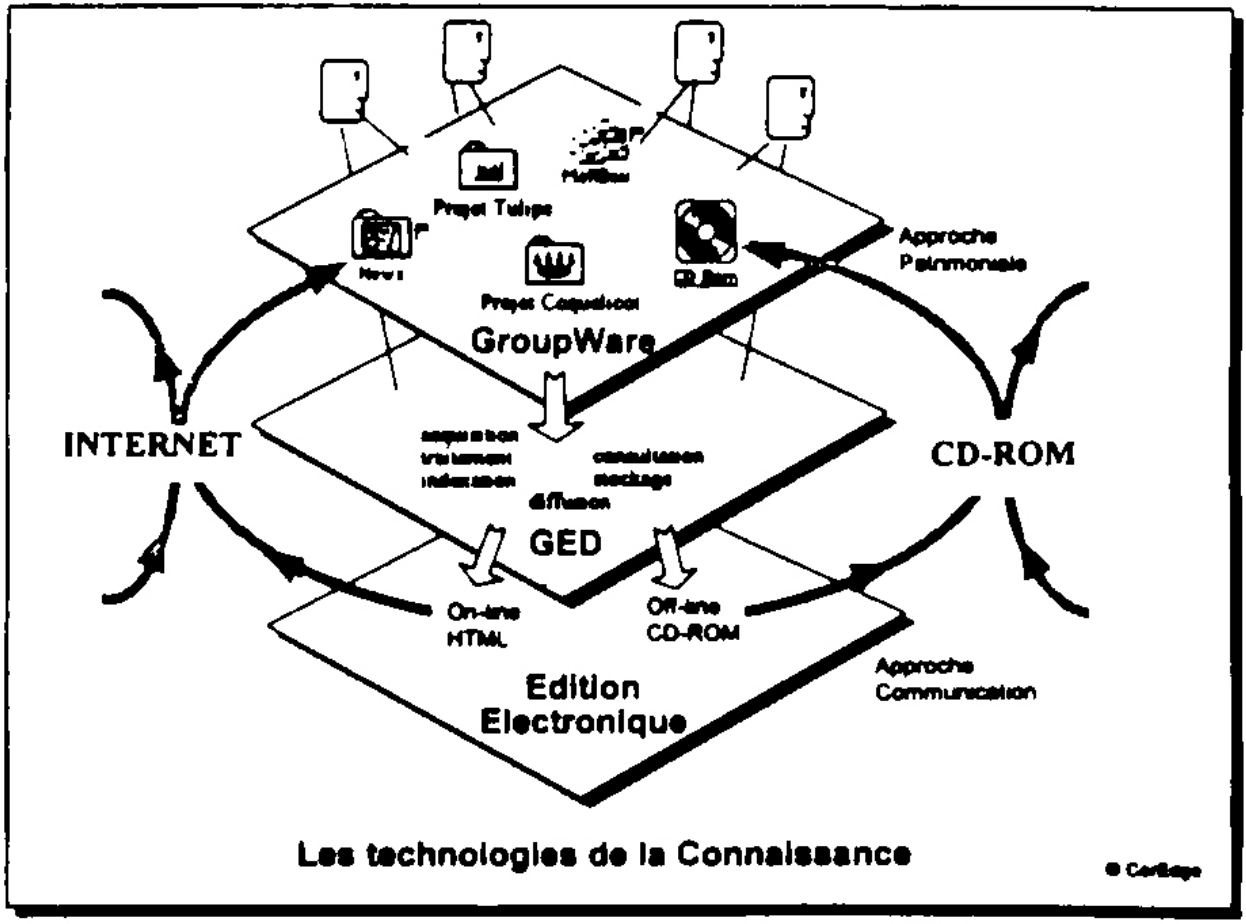

Parcourons ce modèle de bas en haut, en suivant une chaîne de formalisations successives, allant du plus tacite vers le plus formel, du plus contextué vers le plus référentiel :

Niveau de la production collaborative de connaissance

La couche supérieure du modèle fournit au groupe de travail un outil pour faciliter la communication et l'échange d'idées, d'informations, de documents. L'outil participe à la transformation d'une somme de connaissances tacites individuelles (les petits bonhommes dessinés en haut) à une connaissance formalisée collective et éventuellement classée par thème, par projet (les petits dossiers). Si cet échange doit se faire de manière plutôt discrétionnaire, sur un mode forum, l'outil approprié sera le $\mathrm{BBS}^{6}$ ou les forums de messagerie ; si l'échange obéit à des règles et des procédures fixes, alors l'outil sera un workflow.

Le Groupware apportera un soutien efficace à l'élaboration collective, à la communication, la coordination et la coopération des équipes géographiquement dispersées et sur un mode asynchrone.

Hélas, si la construction de connaissance collaborative se fait très bien au sein du groupe de travail, elles est d'un usage limité car :

88 - la connaissance collective dépasse difficilement le périmètre des acteurs du projet (connivence, jargon, hyperspécialisation...),

89 - la connaissance échangée est très liée au contexte spécifique du projet,

90 - elle fait rarement l'objet d'un effort de capitalisation : lorsque le projet s'arrête et les acteurs sont disséminés, il y de gros risques de perte et d'oubli. 


\section{Niveau de la capitalisation} situations. stockages...

\section{Niveau de la diffusion} l'information. (hypertexte) et le feed-back.

Détaillons ces trois points : d'attention; l'intelligence collective ;

\section{Retour à la case départ}

Pour remédier à ces difficultés, la deuxième étape est un travail de capitalisation ; c'est d'abord un travail humain (expert, responsable de domaine, documentaliste) qui consiste à collecter, filtrer, trier, indexer des documents, informations, dans le but de les rendre référentiels. Ensuite les technologies de la GED peuvent aider à rendre ce patrimoine accessible avec pertinence et rapidité à l'ensemble de l'organisation.

La GED répond à cet objectif qui est de permettre à l'utilisateur de retrouver l'information pertinente qui se dissimule au sein des données ${ }^{7}$, indépendamment des acteurs et des

À cet effet la GED met en œuvre des descripteurs (structurés, en texte intégral, par concept, par hyperliens...) et un arsenal de quincaillerie: scanners, compressions,

de la couche Groupware à la couche GED nécessite un travail important et complexe d'analyse linguistique et sémiologique (analyse des corpus et clusters sémantiques, thésaurus, ajustement du niveau d'indexation générique / spécifique).

Les deux premières étapes s'inscrivaient dans une démarche " pull » pour l'organisation, l'aidant à construire puis à préserver et rendre accessible son capital de connaissance.

Mais pour accéder à une information dans une base, encore faut-il la rechercher !

Pour passer dans une logique "push », c'est-à-dire «apprenante » l'organisation doit faire un effort d'éditorialisation («porter à connaissance», pédagogie, diffusion) de

C'est le domaine de prédilection des nouveaux médias électroniques: Web Internet ou Intranet, CD-ROM, qui se démarquent des médias traditionnels d'édition (livre, audiovisuel) par trois caractéristiques : l'interactivité, un mode de lecture navigationnel

1. une entreprise, un hôpital, un Airbus... n'ont pas le temps d'utiliser un média linéaire, comme le livre ou la vidéo, il leur faut un outil permettant d'aller immédiatement au sujet

2. la navigation hypertextuelle fonctionne comme la pensée, sur un mode hologrammatique, où tout nœud ou lien est indissociable de tout un réseau auquel il est connecté; c'est là que peuvent se partager les intersubjectivités et s'élaborer

02 3. Le réseau on-line est le premier média offrant au lecteur la possibilité de rétroaction: libéré de son rôle passif de consommateur subissant une pollution informationnelle, le lecteur adopte une démarche active et participative.

3 En fin de parcours, le produit le plus achevé de notre travail de formalisation électronique, que ce soit un serveur Web ou un CD-ROM, n'est qu'une étape ; il peut être 
"publié » au sein d'un forum Groupware, engendrant ainsi de nouvelles discussions, de nouveaux échanges, ou servant de base au fonctionnement de nouvelles équipes: c'est cette récursivité infinie et dynamique du plus tacite au plus formel, et du formel qui fabrique en retour du tacite, qui nous autorise à conférer au modèle la qualité d'infrastructure à l'ingénierie de la connaissance.

\section{Intranet, un outil fédérateur}

104 Il faut souligner que ces trois niveaux sont contenus dans le champ fonctionnel de l'INTERNET-INTRANET :

105 - les newsgroups et la messagerie (notre couche 1),

106 - les fonctions de recherche (agents intelligents, surindexation), l'interface avec les bases de données et applications via les scritps Java ou ActiveX, liens OBDC (couche 2),

107 - le Web et l'édition de pages HTML (notre couche 3).

\section{Le niveau méthode : piloter le changement}

Les premières expérimentations d'implémentation de NTIC dans des organisations complexes ont mis en évidence un nombre de facteurs jouant un rôle de catalyseur ou inhibiteur dans le processus de changement, que l'on peut résumer par le tableau suivant :

\section{Tableau 2 : catalyseurs et inhibiteurs du changement technologique}

\begin{tabular}{|c|}
\hline $\begin{array}{l}\text { Catalyseurs techniques: } \\
\text { - tendance coût-performance des technologies favorables } \\
\text { - inierconnectivité des réseaux et des plates-formes } \\
\text { - stabilisation des standards (OS, normes de protocoles ISO, CCITT...) } \\
\text { - integration des NTIC avec la Bureautique et les applications de produc- } \\
\text { tivite personnelle } \\
\text { - apparition du document "multimédia " } \\
\text { - aptitude à gérer l'information "non structurée " }\end{array}$ \\
\hline $\begin{array}{l}\text { Inhibiteurs techniques : } \\
\text { - rareté des competences techniques } \\
\text { - manque de vision sur les evolutions futures des NTIC } \\
\text { réduction future des ratios coût-performance (attentisme) } \\
\text { obsolescence rapide } \\
\text { - coût del'integration } \\
\text { inexperience }\end{array}$ \\
\hline $\begin{array}{l}\text { Catalyseurs organisationnels: } \\
\text { - possibilité de mise en place localisée facilitant la mesure de l'efficience } \\
\text { avec une perturbation minimale du fonctionnement } \\
\text { - effet structurant et dynamisant pour la vision stratégique et l'organisa- } \\
\text { tion humaine }\end{array}$ \\
\hline $\begin{array}{l}\text { Inhibiteurs organisationnels: } \\
\text { - manque de vision stratégique } \\
\text { - répugnance a admettre le role stratégique de l'informatique... } \\
\text { inertie de l'organisation } \\
\text { - redististrution des "pouvoirs", conflits centralisation/décentralisation } \\
\text { - coût de transformation important pour faibles economies d'échelle } \\
\text { - inexpérience de pilotage }\end{array}$ \\
\hline $\begin{array}{l}\text { Catalyseurs du marché } \\
\text { - avantage concurrentiel } \\
\text { - avantage coopératif } \\
\text { - pression de la concurrence } \\
\text { - offre des "applications verticales" : revue de presse, gestion de RH, pho- } \\
\text { tothèque... } \\
\text { - services à valeur ajoutée à base de NTIC }\end{array}$ \\
\hline $\begin{array}{l}\text { Inhibiteurs du marché } \\
\text { - ignorance des possibilités offertes par les NTIC et de l'offre } \\
\text { niveau insuffisant des utilisateurs } \\
\text { approche trop conservatrice des entreprises } \\
\text { - offre et demande trop fragmentées et mal ajustées } \\
\text { - trop forte domination du secteur public } \\
\text { saturation médiatique ou publicitaire et réaction aux phénomènes de } \\
\text { mode }\end{array}$ \\
\hline
\end{tabular}




\section{De la conception à l'utilisation}

Il nous faut admettre que le processus de conception et d'appropriation d'un nouveau produit n'est pas linéaire mais constitue un double boucle rétroactive, schématisée cidessous :

Figure 5 : double cycle « conception-utilisation » d'un nouveau produit

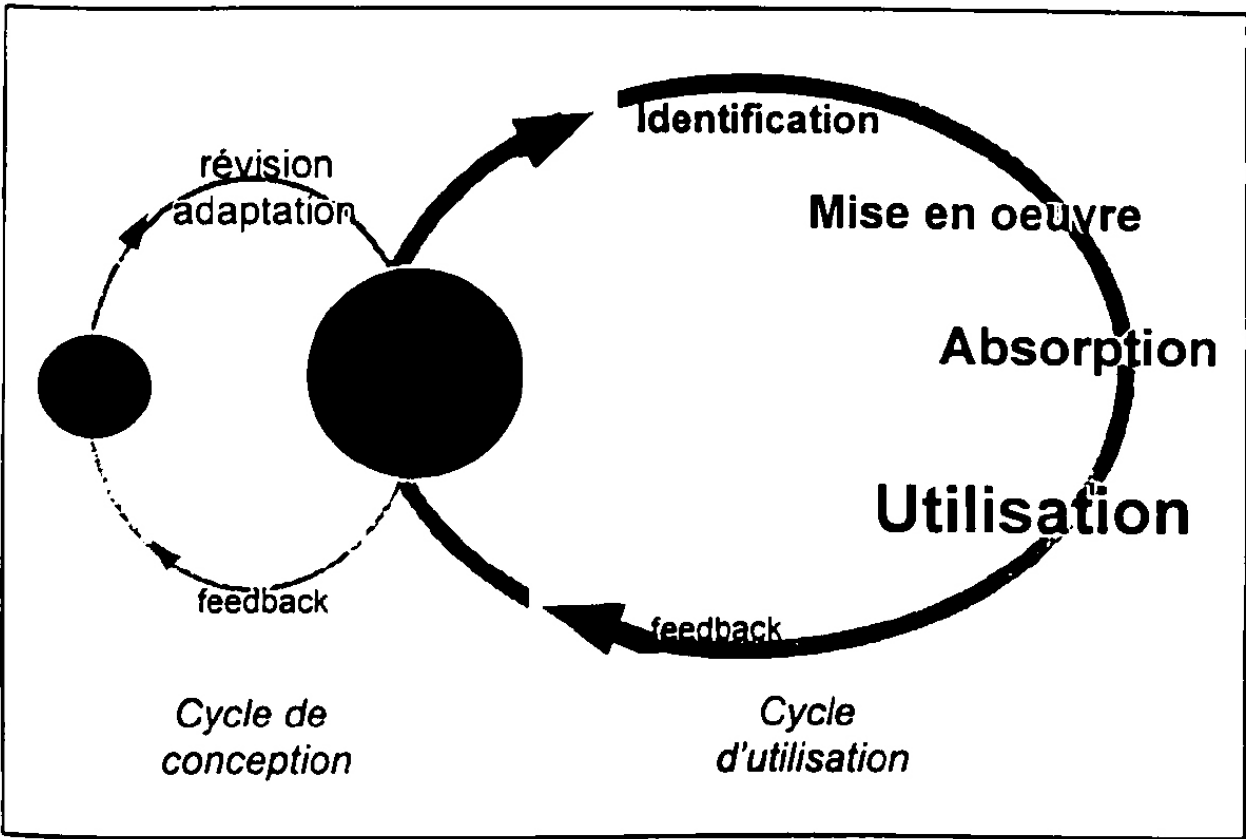

110 Sur cette figure, on voit que les concepteurs des système innovants ont en permanence besoin du feed-back du marché, pour mieux adapter leurs produits aux besoins et réorienter leurs directions de recherche, $\mathrm{n}$ remarque que :

111 - l'utilisateur ne peut pas exprimer des besoins par rapport à des usages qu'il ne connaît pas encore,

112 - dans un contexte d'innovation, le feed-back est toujours très faible, ce qui constitue un facteur de ralentissement et un mauvais ajustement de l'offre avec la demande. En d'autres termes, les méthodes traditionnelles d'«étude de marché » et de «benchmarking " ne fonctionnent pas avec un produit innovant,

113 - si le produit implique un forte interdépendance d'acteurs, il y a risque de cercle vicieux (tout le monde attend tout le monde).

\section{Le niveau stratégie : anticiper et modéliser les connaissances. Introduction au knowledge management}

"Si vous opposez une personne performante à un système déficient, le système gagnera à tous les coups » Mary L. Broad, 1998.

114 Un encouragement à la publication systématique des bonnes pratiques (best practices) et des dysfonctionnements serait porteur de nombreux avantages : 


\section{Bonnes pratiques}

115 Une bonne pratique est une histoire courte racontant la résolution d'un problème, la mise en œuvre d'une idée qui a amélioré le système...

116 On y trouve beaucoup d'avantages :

117 - l'histoire est très contextuée, racontée dans des termes simples et compréhensibles par les « pairs » : elle est plus facilement appropriable ;

118 - l'histoire est très directement liée à une action ou à une activité ; elle évite par conséquent le travail de «traduction » du concept à la pratique ;

119 - l'histoire est portée par des hommes : elle est porteuse de reconnaissance ;

120 - c'est un habitude qui existe mais malheureusement limitée au cercle physique et familier (autour du café ou de la photocopieuse) ;

121 - c'est un moyen de permettre à tous les acteurs de devenir créateurs de contenu.

\section{Dysfonctionnements}

122 La publicité autour des dysfonctionnements et des problèmes n'est pas intégrée à la culture occidentale (complexe de la « faute » judéo-chrétien). C'est fort dommage, car une transparence sur les dysfonctionnements aurait beaucoup d'avantages :

123 - la publication en un point $\mathrm{X}$ d'un dysfonctionnement permettrait d'éviter qu'il ne se reproduise en d'autres points $\mathrm{Y}$ ou $\mathrm{Z}$;

124 - on n'apprend pas mieux que par l'erreur ;

125 - une vue «méta» sur une population d'incidents et d'actions curatives, a priori singuliers, peut aider à dégager une symptomatologie ${ }^{8}$ et donc une optimisation.

\section{Les nouveaux métiers d'intermédiation de l'information}

Depuis trente ans, l'architecture à la base de tous les systèmes d'information était une architecture de type Requête-Réponse (R\&R pour Request \& Reply) : un utilisateur ou une application émet une requête, un producteur ou une routine émet une réponse.

Figure 6 : Architecture classique $R \& R$

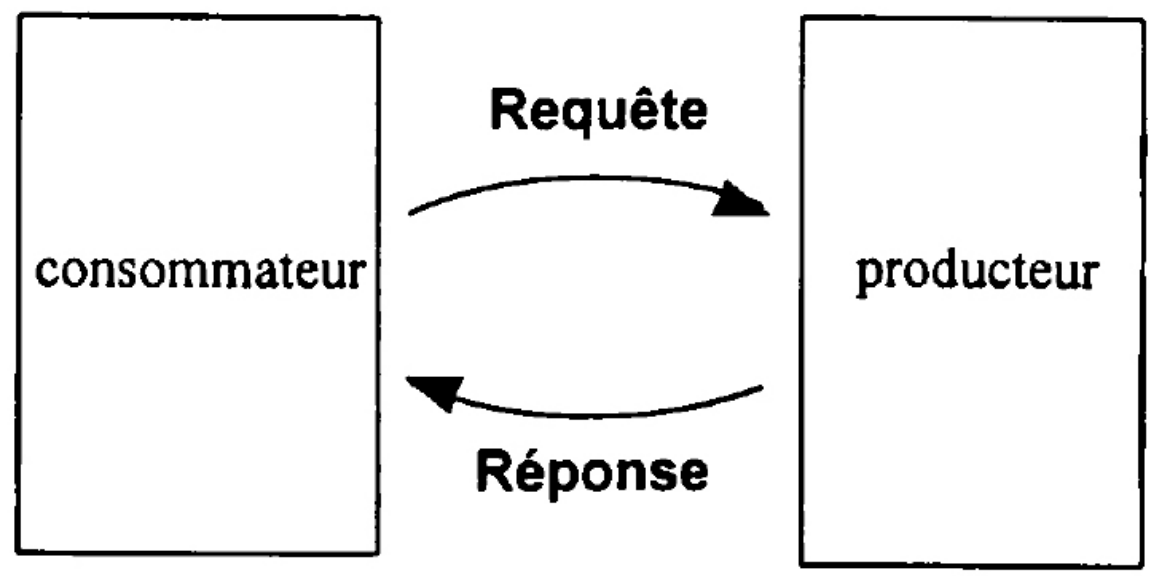



simple, à travers une structure de données unique et figée. Certes, cela convient pour certaines applications simples et rationnelles (fichier client, tableaux de bord de suivi d'activité...), mais pas pour des activités de veille ou d'intelligence économique (tendances émergentes du marché, nouveaux entrants, données «floues» ou « émergentes »...);

132 - dans un monde de globalisation et d'interdépendance, et où producteurs et consommateurs apparaissent et disparaissent fréquemment, il est de plus en plus difficile d'exercer un contrôle sur l'ensemble des flux et des acteurs ;

133 - un système R\&R convient à un modèle transactionnel mais pas à un besoin de coordination de multiples acteurs intervenant dans un processus à la fois comme consommateurs et producteurs d'informations ;

134 - si un producteur veut émettre une information vers des consommateurs qu'il ne connaît pas, il doit recourir à la formule du broadcasting (envoi à tous), qui est peu efficace (forte charge des réseaux pour faible rendement, pollution informationnelle).

Ce dernier point est capital et permet d'introduire la notion de Pull-Push.

\section{La notion de Pull-Push} GED, classeur ou bibliothèque) pour en tirer l'information recherchée. C'est le concept pull9. Il suppose une bon système de repérage de l'information disponible.

138 - « je ne sais pas que je ne sais pas ». Dans ce cas, c'est au système que revient la charge de forcer mon attention pour m'informer de quelque nouvelle que je ne cherchais pas. C'est le concept push, qu'on peut traduire par " porter à connaissance ».

\section{L'intermédiation de l'information}

139 Une architecture nouvelle, bien qu'héritée d'un concept centenaire, a émergé ces dernières armées, qui permet de coordonner et d'animer les différentes composantes d'un système distribué d'information et de communication sans pour autant contrôler les acteurs, les données, les traitements et la présentation. 


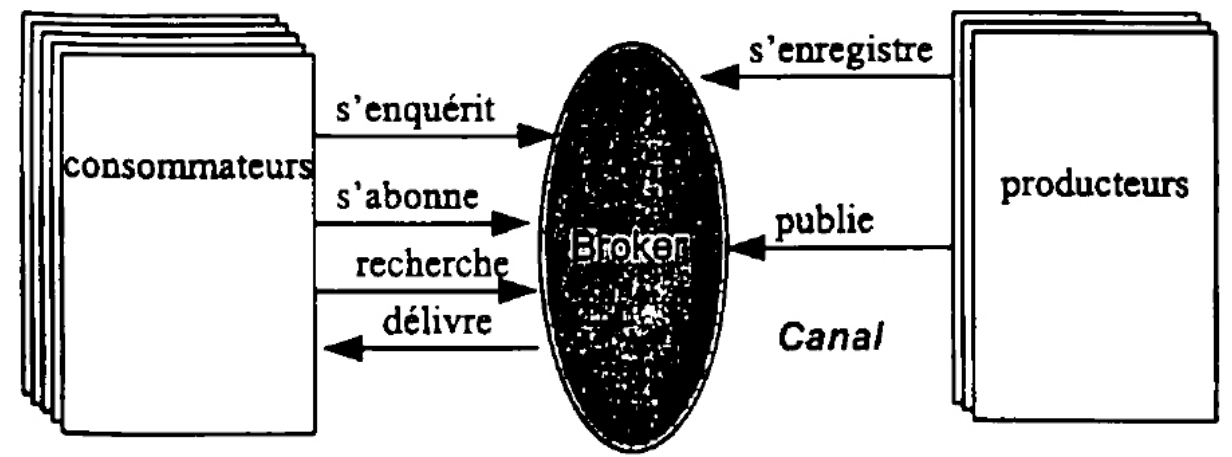

Le principe est de séparer les rôles du producteur et du consommateur en créant une fonction d'intermédiation, que les anglo-saxons nomment broker ${ }^{10}$.

Le broker s'occupe des interactions de sorte que ni le producteur ni le consommateur n'ont besoin de se connaître pour que le système fonctionne. Un lien est établi entre le producteur et le consommateur, il sera maintenu par le broker aussi longtemps que le producteur continuera à produire de l'information et que le consommateur en demandera, c'est-à-dire quelques secondes ou plusieurs années.

Figure 8 : Système distribué d'information et de communication

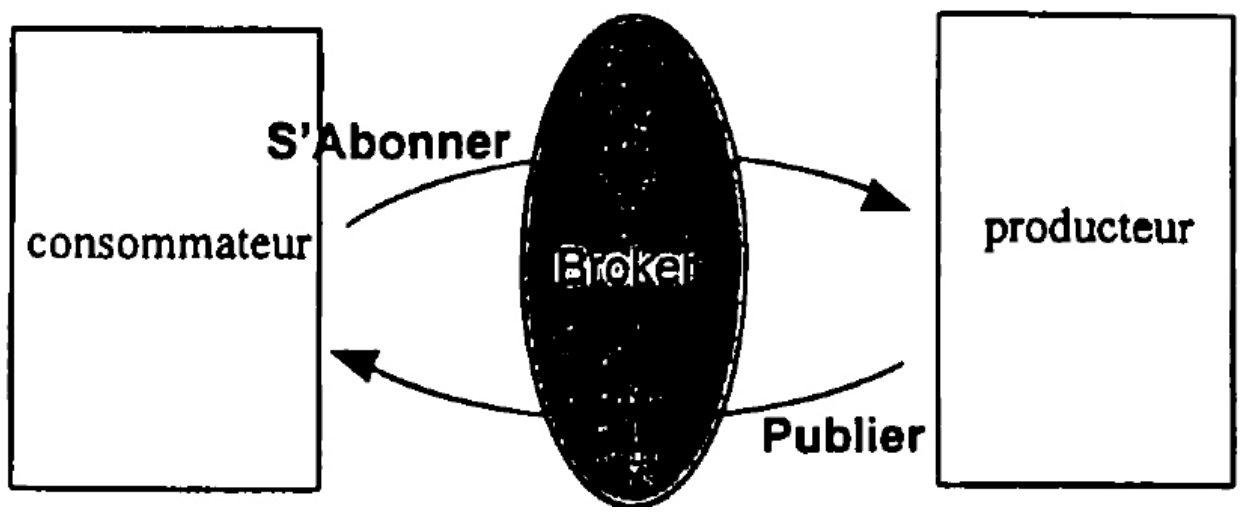

\section{Les nouveaux métiers}

Dans un système de capitalisation de connaissance à l'échelle de la firme, l'intermédiation de l'information, c'est-à-dire la mise en relation de l'offre et de la demande va jouer un rôle déterminant; cela suppose de créer de nouveaux métiers: Webmaster, Knowledge Manager...

Le Webmaster est chargé d'animer un site et un ensemble de forums et d'en garantir la qualité, selon les règles de la charte de certification; il travaille à proximité des producteurs et des consommateurs et répond au Chef de Centre et au Knowledge Manager. C'est un chef de projet polyvalent qui doit cumuler des qualités relationnelles, d'écoute, de travail en équipe avec un goût pour les fonctions de veille et de capitalisation, une bonne aptitude au management et à l'animation. 
Le Knowledge Manager évangélise sur l'importance stratégique du Knowledge Management, et conduit le changement culturel ; il établit la vision globale du système de KM (architecture, organisation, contenu, technique, ressource...) c'est-à-dire une cartographie des compétences; il fixe les principales priorités parmi tous les champs de connaissance, il manage les accès externes du KMS (veille, intelligence économique, volet sécurité), il fixe les exigences de qualité du système, il désigne et missionne les différents partenaires du projet, notamment les coordonnateurs.

\section{Conclusion : manager la connaissance dans l'entreprise}

L'ingénierie de la connaissance collective doit s'inscrire dans une vision constructiviste : la connaissance n'est qu'une construction et non le miroir objectif d'une réalité; l'information est la construction d'une représentation, la communication a pour objet de faire partager et ajuster les représentations.

146 La mise en place d'outils et de supports d'échanges n'est jamais neutre : le fait de coucher sur le papier une pensée transforme cette pensée, le fait de retracer un événement modifie notre analyse de cet événement. La structuration des idées échangées joue un rôle déterminant sur la productivité des échanges, et, par là, sur l'innovation dans le mode de fonctionnement des réseaux.

147 La mise en place d'un dispositif de capitalisation de connaissance à l'échelle de la firme est un enjeu stratégique majeur qui devrait modifier profondément la relation de l'homme à la connaissance et au travail. Ce dispositif devient alors :

148 - un facteur de développement social : la structuration du travail en groupe et de l'entreprise en réseau, basée sur la reconnaissance mutuelle de l'apport des compétences à une finalité partagée et non sur la seule hiérarchie, améliore le sentiment personnel et mutuel d'appartenance et de valorisation;

149 - un facilitateur des organisations par processus transverses: la qualité client, la performance et la compétitivité, la production de valeur seront améliorées par la communication, la coordination et la coopération entre les équipes ;

150 - un facilitateur de l'apprentissage collectif : l'amélioration des performances et de l'expertise individuelles et d'équipe, le développement de la réactivité et de l'anticipation, l'innovation par hybridation, concourent à une organisation générant « un tout supérieur à la somme des parties ".

\section{BIBLIOGRAPHIE}

BAUMARD Philippe, Les organisations déconcertées; gestion stratégique de la connaissance, Éditions Masson, 1996.

GENELOT Dominique, Manager dans la complexité, INSEP Éditions, 1992.

NonAKA Ikujiro et TAKeUCHI Hirotaka, The Knowledge Creating Company, Oxford University Press, 1995, New York.

Communication et organisation, 13 | 1998 
PRAX Jean-Yves, Manager la connaissance dans l'entreprise, INSEP Éditions, 1997.

PRAX Jean-Yves, La gestion électronique documentaire, Armand Colin, 1993 (réédition sous-presse Dunod-InterEditions).

\section{NOTES}

1. GENELOT Dominique, Manager dans la complexité, INSEP Editions. 1992

2. NONAKA I., A dynamic theory or organizational knowledge creation - Organization Science, vol5, $\mathrm{n}^{\circ} 1, \mathrm{p} 88-102,1969$.

3. Idem.

4. AUMARD Philippe, Les organisations déconcertées. La gestion stratégique de la connaissance, Masson, 1996.

5. PRAX Jean-Yves. « Manager ta connaissance dans l'entreprise » INSEP Editions, 1997

6. BBS : Bulletin Board System, Babillard électronique en français.

7. PRAX Jean-Yves, « La Gestion Electronique Documentaire », Armand COLIN, 1993.

8. On peut se référer à l'histoire, souvent tristement célèbre, des grandes épidémies.

9. Celui des agences de presse.

10. Le broker est celui qui achète en gros pour revendre au détail. Il connaît les besoins spécifiques de ses clients et est capable de les grouper pour avoir du poids sur les offreurs. Les termes français seraient médiateur ou agent ; cependant Us risquent de porter i confusion.

\section{RÉSUMÉS}

Face à un environnement complexe, incertain et hautement concurrentiel, l'entreprise qui veut rester compétitive doit mobiliser son intelligence collective et manager sa connaissance.

Cela suppose des dispositifs répartis sur l'ensemble des acteurs, qui posent à l'entreprise des défis culturels, organisationnels et stratégiques majeurs.

Les nouvelles technologies de l'information et de la communication, en facilitant le partage et la coordination entre les acteurs, donnent une réponse nouvelle à cette problématique d'ingénierie de la connaissance collective.

Dans cet article, l'auteur aborde dans un premier temps le concept de connaissance organisationnelle (qu'est-ce que c'est? À quoi cela sert?)

Puis il s'appuie sur des retours d'expériences concrètes pour proposer une méthodologie d'ingénierie de la connaissance (comment s'y prendre ?) basée sur une approche à trois niveaux : outil, management, stratégie.

Companies that wish to keep alive within a highly complex, impredictible and competitive environment, must manage their organizational knowledge-

It supposes the ability to set up a knowledge capturing System that actually involves all the players. It supposes also to adress successfully the cultural and organizational obstacles that such a change usually induces.

New Technologies of Information and Communication can open the way to collaborative strategies based on invention, intelligence and knowledge sharing. 
First, the author defines the concept of organizational knowledge (what is it ? What is the use of it?). Second, based on real experiences, he proposes a new method for the collaborative knowledge engineering (how to manage it?) structured in three levels: tools, management, strategy.

\section{AUTEUR}

\section{JEAN-YVES PRAX}

Jean-Yves Prax, Docteur ès Sciences, est Président Directeur Général d'INSEP COREDGE. Son expertise du Knowledge Management et des NTIC est sollicitée par les grandes entreprises, par des organismes tels que l'ANVAR, la COB, la CEE et par les organisateurs de conférences. Il est auteur de plusieurs livres sur le sujet et est chargé d'enseignement en DESS Sciences Politiques et en Magistère de Gestion à Paris I-Sorbonne. 